\title{
ARTICLE
}

\section{Interpreting standardized and idiographic outcome measures in CAMHS: What does change mean and how does it relate to functioning and experience?}

\author{
Julian Edbrooke-Childs \\ Jenna Jacob \\ Duncan Law \\ Jessica Deighton \\ Miranda Wolpert
}

\section{Declaration of Interests}

Julian Edbrooke-Childs and Jessica Deighton are members of the Policy Research Unit in the Health of Children, Young People and Families (CPRU). Miranda Wolpert is a paid director (1 day a week) of the Child Outcomes Research Consortium (CORC) and is the lead of the mental health stream of CPRU. Jenna Jacob is a current member of the CORC central team. No other authors have any conflicts to declare.

\section{Acknowledgement}

The Policy Research Unit in the Health of Children, Young People and Families is funded by the Department of Health Policy Research Programme. This is an independent report commissioned and funded by the Department of Health. The views expressed are not necessarily those of the Department. The authors would like to thank members of CPRU: Terence Stephenson, Catherine Law, Amanda Edwards, Ruth Gilbert, Steve Morris, Helen Roberts, Cathy Street, and Russell Viner. The authors would also like to thank all members of CORC and its board and committee at the time of writing-(including M.W. and D.L.): Ashley Wyatt, Alison Towndrow, Tamsin Ford, Julie Elliott, Ann York, Mick Atkinson, Kate Martin, Neil Humphrey and Alan Ovenden-and the CORC central team at the time of writing (including J.J.): Isobel Fleming, Elisa Napoleone, Victoria Zamperoni, Christa Daboiko, Charlotte Payne, Kallum Rogers, Kate Dalzell, Craig Hamilton, Sally Wilson, Mark Garbett, Deborah Sheppard, Alison Ford, Amy Ellis-Thompson and Jeni Page. 


\begin{abstract}
Patient Reported Outcome Measures (PROMs) are increasingly being used in Child and Adolescent Mental Health Services (CAMHS). The aim of the present research was to explore change in standardized and idiographic outcome measures in CAMHS using naturalistic, routinely collected data. We explored change in psychosocial difficulties and impact on daily life as evaluated by a broad standardised measure, the Strengths and Difficulties Questionnaire (SDQ) (Goodman, 1997), and progress toward goals as evaluated by a personalised idiographic measure, the Goal Based Outcomes tool (GBO) (Law, 2011) in a sample of $N=137$ CAMHS attenders. Psychosocial difficulties and impact on daily life showed less change over the course of treatment than progress toward goals in the present study. Change in psychosocial difficulties and impact on daily life also showed fewer significant associations with change in clinician-reported functioning and satisfaction with care at time two than change in progress toward goals. Findings of the present research may support previous studies in which service users and clinicians report that idiographic measures are more capable than standardised measures of capturing relevant change for individuals.
\end{abstract}

\title{
Key Practitioner Message
}

- For normative comparison and clinical purposes, it is important to know how to interpret change in standardized and idiographic measures in CAMHS.

- Past research suggests that standardized measures (e.g., the Strengths and Difficulties Questionnaire or SDQ) may be less clinically useful than idiographic measures (e.g., the Goal Based Outcome tool or GBO), but more amenable for aggregation and therefore comparing groups of patients.

- The SDQ showed less change than the GBO in the present study in terms of mean score change and reliable change.

- Change captured by the SDQ was less strongly associated with change in clinicianreported functioning and satisfaction with care at time two than change captured by the GBO, and only change in the GBO was uniquely associated with change in clinicianreported functioning over treatment.

- Findings of the present study suggest that change greater than 2.45 points in the GBO represents reliable change.

Keywords: Mental Health, Child, Adolescent, Goals, Questionnaires 
Routine outcome monitoring is "the systematic evaluation of a patient's treatment response during the course of treatment and provides health care professionals with information relevant to a patient's progress" (Carlier et al., 2012, p. 104). It is recommended by healthcare systems internationally and serves two main purposes (Department of Health, 2012; National Quality Forum, 2013).

The first purpose is to provide clinicians with useful information about what treatments are working, or not working (Lambert et al., 2006; Whipple \& Lambert, 2011). Feedback theory states that when individuals are presented with a discrepancy between their actual performance and their standards for performance, they are motivated to reduce the discrepancy (Kluger \& DeNisi, 1996; Sapyta, Riemer, \& Bickman, 2005). Evidence suggests that routine outcome monitoring has a positive impact on treatment outcome and that children improve faster when their clinician receives this feedback (Bickman, Kelley, Breda, de Andrade, \& Reimer, 2011; Carlier, et al., 2012; Kelley \& Bickman, 2009; Knaup, Koesters, Schoefer, Becker, \& Puschner, 2009; Lambert \& Shimokawa, 2011). Routine outcome monitoring dovetails with policy on increasing service user involvement in care (Department of Health, 2010) as it facilitates patient-clinician communication, enabling patients to collaborate in treatment decisions (Carlier, et al., 2012; Chen, Ou, \& Hollis, 2013) .

The second purpose is that it may provide information useful for service evaluation. Information obtained from routine outcome measures may be used by clinicians and supervisors to review patients' treatment progress and discuss areas for change, especially when patients may not be responding to treatment as expected (Law, 2011). It may be used by a service as a whole or in conversations about service funding with commissioners (Wolpert et al., 2014).

A number of key performance indicators and targets exist for English child mental health services about the return rate of, and change observed in, outcome measures (NHS Institute for Innovation and Improvement, 2013). For clinical and service evaluation purposes, it is important to know how to interpret changes in outcome measures.

In Child and Adolescent Mental Health Services (CAMHS) in England, patient reported outcome measures (PROMs), clinician reported outcome measures, or patient reported experience measures are collected. PROMs may comprise standardized or idiographic measures. Although standardized measures may be more useful for comparing change and aggregating information for groups of patients, these measures may be perceived as lacking clinical utility and not representing the individual (Bromley \& Westwood, 2013; Norman, Dean, Hansford, \& Ford, 2013; Ruble, McGrew, \& Toland, 2012).

It has also been argued that standardised measures focus on symptoms and neglect other domains such as coping skills, which are particularly pertinent when working with children with conditions where symptoms may not improve (Batty et al., 2013). One example of a widely used standardized measure is the Strengths and Difficulties Questionnaire (SDQ; Goodman, 1997), which is a measure of psychosocial difficulties and their impact on daily life.

Idiographic measures are selected on the basis of the individual's specific difficulties, or the area they and the clinician feel should be the focus of treatment. Service users and clinicians may see these as more capable than standardised measures of capturing relevant change (Bromley \& Westwood, 2013; Norman, et al., 2013; Pender, Tinwell, Marsh, \& Cowell, 2013). However, it has been argued that the idiosyncratic nature of such measures makes them more vulnerable to subjective interpretation, especially if performance targets are introduced (Bevan \& Hood, 2006; Law, 2011). A widely used idiographic measure in child mental health services in England is the Goal Based Outcomes measure (GBO). Clinicians, children, and parents - independently or, ideally, jointly - agree upon their goals and rate progress towards achieving these over the course of treatment (Law, 2011).

Setting explicit goals makes the process of achievement more salient by highlighting intermediate tasks necessary to final achievement (Austin \& Vancouver, 1996; Goodman, 2001; Karoly, 1993; Locke \& Latham, 1990). Setting and monitoring progress may improve patient activation, meaning that patients are better able to self-manage health conditions 
(see Hibbard \& Gilburt, 2014). Almost $90 \%$ of clinicians agree that goal setting helps to structure and focus treatment (Pender et al., 2013).

Young people report that goals help to prioritise the target of treatment (Bromley \& Westwood, 2013) although they may be concerned that goal achievement will result in treatment termination (Moran, Kelesidi, Guglani, Davidson, \& Ford, 2011). Young people have been shown to set three broad types of goals in CAMHS: relationship/interpersonal, coping with specific problems and symptoms, and personal growth and functioning (Bradley, Murphy, Fugard, Nolas, \& Law, 2013).

Other goal setting outcome measures such as Goal Attainment Scaling (GAS) (Kiresuk \& Sherman, 1968) and Target Complaints (Battle, Imber, Hoehn-Saric, Nash, \& Frank, 1966) are widely used in healthcare (e.g., Maher \& Barbrack, 1984; Ruble et al., 2012). Reviews of these measures have found evidence to support their psychometric properties, especially in terms of validity (Donnelly \& Carswell, 2002; Hurn, Kneebone, \& Cropley, 2006). Goal Attainment Scaling is widely used in child physical health settings (Steenbeek et al., 2011) and to a lesser extent in child mental health settings, in particular with children with conduct disorder (Maher \& Barbrack, 1984) and autistic spectrum disorder (Ruble et al., 2012).

To the best of our knowledge, no existing study has explored change in psychosocial difficulties, impact on daily life, and progress toward goals in CAMHS in England using naturalistic, routinely collected data. Evidence is needed regarding what these changes mean and how they should be interpreted.

\section{Aim of the Present Research}

The aim of the present research was to explore change in standardized and idiographic outcome measures in CAMHS using naturalistic, routinely collected data. We expected that progress toward goals would show greater change associated with treatment than psychosocial difficulties and impact. In addition, we expected that change in progress toward goals would show more associations with change in clinician reported outcome measures and patient reported experience measures.

\section{Method \\ Participants and Procedure}

The Child Outcomes Research Consortium (CORC) is a professional learning collaboration whose members provide services for children and young people with mental health difficulties and collect data to inform clinical practice and service improvement (CORC, 2014b).

Children from this dataset were included if their data were reported in or later than 2007, and if they had complete progress towards goals, psychosocial difficulties, and impact on daily life (see Measures). This resulted in a final sample of $N=137$ (50\% female, $46 \%$ male, $4 \%$ not specified; $40 \%$ aged $0-12$ and $60 \%$ aged $13-18$ ) children from 14 services. The age and gender demographics in the present dataset were consistent with the demographics in an Office for National Statistics survey of the prevalence of childhood mental health problems (Green, McGinnity, Meltzer, Ford, \& Goodman, 2005). The measures were taken from a secondary analysis of routinely collected data so ethical review was not relevant (NHS, 2015).

In total, $64 \%$ of children were White, $6 \%$ Mixed, $4 \%$ Asian, 3\% Black, 3\% reporting another ethic group and $20 \%$ not specified or missing;. Previous research has shown that children from black and minority ethnic groups may be under-represented in CAMHS (Malek \& Joughlin, 2004). The presenting problems recorded were emotional disorder (28\%), selfharm $(5 \%)$, conduct disorder (2\%), eating disorders (1\%), hyperactivity $(4 \%)$, autism $(5 \%)$, learning difficulties (6\%), habit disorder (2\%), developmental difficulties (4\%), psychosis $(1 \%)$, and other problems $(7 \%)$ (multiple responses permitted). The demographic characteristics of the present sample seem to be consistent with previous research, which has shown that the majority of children with emotional disorders are likely to be girls in the older age group (Green et al., 2005).

The pattern of presenting problems is also consistent; emotional and behavioural disorders are among the most prevalent. However, the incidence of self-harm was higher in the 
present sample (Green et al., 2005). This may suggest that the present sample is not representative, or it may support anecdotal evidence from CAMHS clinicians that the incidence of self-harm has increased over the past few years (CORC, 2014c).

According to the CORC 'snapshot' protocol, questionnaires are completed by children, parents, and/or clinicians at assessment (Time 1 or T1) and again four to six months later (Time 2 or T2) or if sooner, case closure for all measures with the exception of the SDQ, for which T2 is not collected at case closure (CORC, 2014a).

\section{Measures}

Psychosocial difficulties (T1 and T2). To measure psychosocial difficulties, the 25-item parent-reported Strengths and Difficulties Questionnaire (SDQ) (Goodman, 1997; Goodman, Meltzer, \& Bailey, 1998) was used. The SDQ comprises five subscales assessing psychosocial difficulties, four of which are summed to create the total difficulties score (i.e., conduct problems, emotional problems, peer problems, and hyperactivity). The SDQ is a widely used measure of total difficulties and has demonstrated reliability and validity in numerous studies. The internal consistency of the SDQ has been reported as .82 (Goodman et al., 1998).

Impact on daily life (T1 and T2). To measure impact on daily life, the 5-item parentreported SDQ impact supplement was used. The impact supplement measures overall distress and social impairment, and is scored from not at all (0) to a great deal (2); the internal consistency of the impact supplement has been reported as .85 (Goodman, 2001).

Progress toward goals (T1 and T2). To measure progress toward goals, the 3-item Goal Based Outcome tool (GBO) (Law, 2011) was used. The GBO is an idiographic PROM, and up to three self-determined goals are formulated at the start of treatment, set by the young person, parent, clinician, or ideally jointly agreed. In the present sample, most goals at T1 were mutually agreed $(42 \%)$, with the rest set by the child $(25 \%)$, the parent $(13 \%)$, or the clinician (2\%), with $18 \%$ not specified. Progress toward each goal is then rated on a scale from goal not met at all (0) to goal reached (10). Overall, most progress toward goals at T2 was jointly agreed (39\%), with the rest reported by the child $(26 \%)$, the parent $(19 \%)$, or the clinician (1\%), with $15 \%$ not specified. Three broad themes of child-rated goals have are set in CAMHS (Bradley et al., 2013). The internal consistency in the present study for T1 was .71 and for T2 .73, which are acceptable for research purposes (Nunally, 1967).

Aggregating the three goals was appropriate as the GBO is a measure of overall progress towards therapeutic goals (Law, 2011). Other aspects of goals such as relative importance or anticipated difficulty are not recorded, meaning other types of aggregation were not possible.

Functioning (T1 and T2). To measure functioning, the unidimensional Children's Global Assessment Scale (CGAS; Shaffer et al., 1983) was used. The CGAS is a widely-used measure of global functioning, completed by clinicians on a scale from lowest functioning (1) to excellent functioning (100), which has demonstrated reliability and validity in previous studies (Rey, Starling, Wever, Dossetor, \& Plapp, 1995; Shaffer, et al., 1983).

Satisfaction with care (T2 only). To measure parent-reported satisfaction with care, the Experience of Service Questionnaire (ESQ) (Attridge-Stirling, 2003) was used. The ESQ is a patient-reported experience measure that captures satisfaction with aspects of care received. One subscale was used to assess satisfaction with care received (nine items; e.g., "It was easy to talk to the people who have seen my child") (Brown, Ford, Deighton, \& Wolpert, 2012). Parents responded on a three-point scale from certainly true (1) to not true (3), with a fourth option (don't know) not used in scoring. The ESQ is widely used in CAMHS and has demonstrated reliability and validity (Brown, et al., 2012). As shown in the note of Table 1, internal consistency was .88, which is acceptable for research purposes (Nunally, 1967).

\section{Analytic Strategy}

To examine the relationship between change in psychosocial difficulties, impact on daily life, progress toward goals, functioning, and satisfaction with care, we conducted five analyses. First, we examined change associated with treatment by computing paired samples t-tests to 
examine whether scores in psychosocial difficulties, impact on daily life, and progress toward goals significantly changed between $\mathrm{T} 1$ and T2. Second, we examined reliable change associated with treatment by computing the proportion of cases showing reliable change in psychosocial difficulties, impact on daily life, and progress toward goals between T1 and T2 using the reliable change index (Jacobson \& Truax, 1991). The reliable change index estimates the amount of change required in a measure to conclude that the change observed is not solely attributable to measurement error. Participants can be classified as reliably improved, reliably deteriorated, or not changed. Reliable change indices were calculated using the internal consistencies reported above (see Measures) and T1 standard deviations. A difference of greater than 8.66 was calculated as reliable change for psychosocial difficulties, 1.29 for impact, and 2.45 points for progress toward goals.

Third, to examine the associations between change in psychosocial difficulties, impact on daily life, and progress toward goals with change in functioning and satisfaction with care at T2, we computed bivariate correlations between all variables. For variables completed at T1 and T2, we computed change scores by regressing the T1 score on the T2 score and saving the standardized residual. As functioning and satisfaction scores were missing for some children, these analyses were conducted on a sub-sample $(n=92)$ of children.

Fourth, to examine whether change in progress toward goals showed significantly different associations with change in functioning and satisfaction with care at T2, we computed differences between two dependent correlations (Steiger, 1980). This test compares the correlation between change in goals and change in functioning $(r(\Delta$ progress toward goals, $\Delta$ CGAS)), for example, with the correlation between change in psychosocial difficulties and change in functioning and ( $r(\Delta$ psychosocial difficulties, $\Delta$ CGAS)), producing a $z$-score for the difference, with $z$ greater than $|1.96|$ being significant. Finally, to examine whether change in psychosocial difficulties, impact on daily life, and progress toward goals were uniquely associated with change in functioning and satisfaction with care at T2, we computed hierarchical regressions.

\section{Results}

\section{Change Over Time}

Paired samples t-tests showed that mean scores for psychosocial difficulties, impact on daily life, and progress toward goals increased from T1 to T2, as shown in Table 1. The effect size for psychosocial difficulties was medium, while the effect sizes for impact on daily life and progress toward goals were both large (Cohen, 1988). 
Table 1.

Paired-samples t-test for T1-T2 change in psychosocial difficulties, impact on daily life, and progress toward goals.

Note. $N=137$. T1 = Time $1, \mathrm{~T} 2=$ Time 2 . Higher scores of psychosocial difficulties indicate higher levels of difficulties. Higher scores of impact indicate higher levels of negative impact. Higher scores of progress toward goals indicate higher levels of

Mean $t \quad S D \quad d$

Psychosocial difficulties
1. T1 Difficulties
17.86
7.36
2. T2 Difficulties
14.33
$6.36^{\star * \star}$
0.46
8.03

Impact on daily life

3. T1 Impact

4. T2 Impact

Progress toward goals

$10.22^{\star \star *}$

1.99

2.50

5. T1 progress toward goals

2.25

$20.63^{* * *}$

1.64

6. T2 progress toward goals

6.57

1.99

progress towards achieving goals.

${ }^{\star}=p<.05,{ }^{* \star}=p<.01,{ }^{* \star *}=p<.001$.

\section{Reliable Change}

Overall, most parents (77\%) reported reliable improvement in progress towards goals, followed by impact on daily life (64\%), and then psychosocial difficulties (18\%), as shown in Figure 1. In contrast, most parents reported no reliable change in psychosocial difficulties $(80 \%)$, followed by impact on daily life (29\%), and then progress towards goals $(22 \%)$. Finally, some parents reported reliable deterioration in impact on daily life $(7 \%)$, followed by psychosocial difficulties (2\%) and then progress towards goals (1\%). 


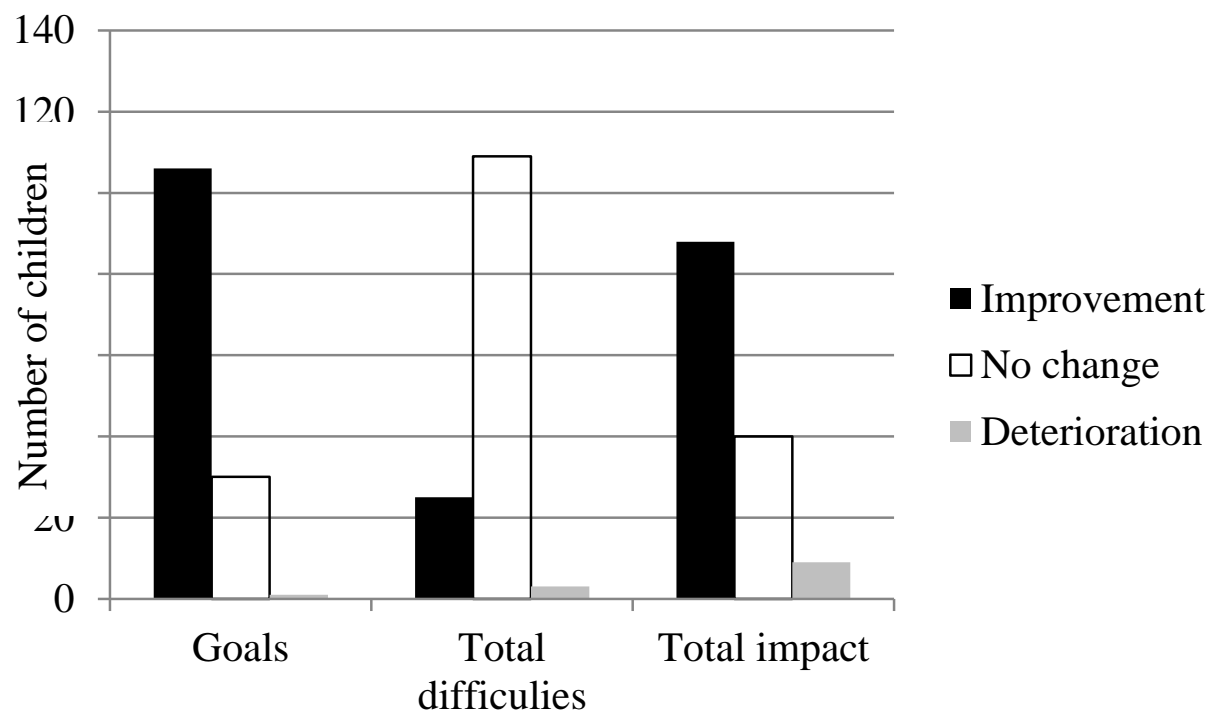

Figure 1.

Reliable change in psychosocial difficulties, impact on daily life and progress toward goals.

Note. $N=137$.

\section{Correlations}

All significant correlations between change scores were in the expected direction, as shown in Table 2. Parents reporting higher levels of difficulties over time also reported higher levels of impact than parents reporting lower levels of difficulties. Moreover, parents reporting these higher levels of difficulties and impact also reported lower levels of progress toward goals over time, functioning over time, and satisfaction with care at T2 than parents reporting lower levels of difficulties or impact over time. Parents reporting higher levels of progress towards goals over time reported higher levels of functioning over time and satisfaction with care at T2 than parents reporting lower levels of progress towards goal. 
Table 2.

Correlations between all variables.

$\begin{array}{llll}1 & 2 & 3 & 4\end{array}$

1. $\Delta$ Psychosocial difficulties

2. $\Delta$ Impact on daily life $.42^{* * *}$

3. $\Delta$ Progress toward goals

$-.39^{\star * \star}-.40^{\star \star \star}$

4. $\Delta$ Functioning

$-.37^{\star \star *}-.34^{\star \star *} \quad .55^{\star * *}$

5. T2 Satisfaction with care

$.26^{*} \quad .28^{\star *}-.24^{*} \quad-.07$

Note. $N=$ 92. $\Delta=$ change (see

Analytic strategy). $\mathrm{T} 2=$ Time 2. Higher scores of psychosocial difficulties indicate higher levels of difficulties. Higher scores of impact on daily life indicate higher levels of negative impact. Higher scores of progress toward goals indicate higher levels of progress toward achieving goals.

${ }^{*}=p<.05,{ }^{* *}=p<.01,{ }^{* * *}=p<.001$.

\section{Differences between correlations}

Tests of the difference between dependent correlations (see Analytic strategy) showed a number of significant differences. The correlation between change in goals and change in functioning $(r(\Delta$ progress toward goals, $\Delta$ CGAS $))$ was stronger than the correlations between change in psychosocial difficulties and change in functioning ( $r(\Delta$ psychosocial difficulties, $\Delta$ CGAS)) with $z=5.90$. The correlation between change in goals and change in functioning $(r(\Delta$ progress toward goals, $\Delta$ CGAS $))$ was stronger than the correlation between change in impact and change in functioning ( $r(\Delta$ impact on daily life, $\Delta$ CGAS) with $z=5.66$. The correlation between change in goals and satisfaction with care at T2 ( $r(\Delta$ progress toward goals, T2 satisfaction with care)) was stronger than the correlation between change in psychosocial difficulties and satisfaction with care at T2 $(r(\Delta$ psychosocial difficulties, $\Delta$ satisfaction with care)) with $z=-2.92$. The correlation between change in goals and satisfaction with care at T2 ( $r(\Delta$ progress toward goals, T2 satisfaction with care $))$ was stronger than the correlation between change in impact and satisfaction with care at T2 $(r(\Delta$ Impact on daily life, $\Delta$ satisfaction with care)) with $z=-3.03$. Notwithstanding, the correlation between change in psychosocial difficulties and change in functioning $(r(\Delta$ psychosocial difficulties, $\triangle$ CGAS)) was not significantly different to the correlation between change in impact and change in functioning ( $r(\Delta$ impact on daily life, $\Delta$ CGAS $)$ ) with $z=-0.29$. Likewise, the correlation between change in psychosocial difficulties and satisfaction with care at T2 ( $r(\Delta$ psychosocial difficulties, T2 satisfaction with care $))$ was not significantly different to the correlation between change in total impact and satisfaction with care at T2 $r(\Delta$ impact on daily life, $\Delta$ satisfaction with care) with $z=-0.18$.

In summary, the relationships between change in progress toward goals and change in functioning, or with satisfaction with care at T2, were consistently stronger than the relationships between change in psychosocial difficulties or impact on daily life. The relationships between change in psychosocial difficulties or change in impact on daily life with change in functioning or satisfaction with care at T2 were not significantly different.

\section{Hierarchical Regressions}

Main effects explained $35 \%$ of the variance of change in functioning and $11 \%$ of the variance of satisfaction with care at T2, while the interaction terms explained no additional variance, as shown in Table 3. Change in goals was the only significant predictor of change in functioning, and parents with higher levels of progress towards goals had higher levels of 
change in functioning than parents with lower levels of progress. Still, no predictors of satisfaction with care at T2 were significant.

Table 3.

Hierarchical multiple regressions predicting change in functioning and satisfaction with care at T2.

\begin{tabular}{|c|c|c|c|c|}
\hline & \multicolumn{2}{|c|}{$\Delta$ Functioning } & \multicolumn{2}{|c|}{ T2 Satisfaction with care } \\
\hline Steps and variables & $\Delta \mathrm{R}^{2}$ & $\beta$ & $\Delta \mathrm{R}^{2}$ & $\beta$ \\
\hline Step 1: Main effects & $.351^{\star \star}$ & & $.112^{*}$ & \\
\hline$\Delta$ Psychosocial difficulties & & -.19 & & .14 \\
\hline$\Delta$ Impact on daily life & & -.08 & & .17 \\
\hline$\Delta$ Progress toward goals & & $.45^{\star \star \star}$ & & -.12 \\
\hline Step 2: Interaction terms & .020 & & .018 & \\
\hline$\Delta$ Difficulties * $\Delta$ Goals & & -.11 & & .05 \\
\hline $\begin{array}{l}\Delta \text { Difficulties }{ }^{*} \Delta \text { Impact } \\
\Delta \text { Goals* } \Delta \text { Impact }\end{array}$ & & $\begin{array}{l}-.13 \\
-.06\end{array}$ & & $\begin{array}{l}-.15 \\
-.11\end{array}$ \\
\hline
\end{tabular}

Note. $N=92 . \Delta$ = change (see Analytic strategy). T2 = Time 2. Higher scores of psychosocial difficulties indicate higher levels of difficulties. Higher scores of impact indicate higher levels of negative impact. Higher scores of goals indicate higher levels of progress towards achieving goals.

${ }^{*}=p<.05,{ }^{* *}=p<.01,{ }^{* * *}=p<.001$.

\section{Discussion}

The aim of the present research was to explore change in standardized and idiographic outcome measures in CAMHS using naturalistic, routinely collected data. We explored change in psychosocial difficulties, impact on daily life, and progress toward goals. In line with expectations, psychosocial difficulties and impact on daily life showed less change than progress toward goals in the present study. Over the course of treatment, mean psychosocial difficulties scores decreased with a medium effect size, mean impact on daily life scores decreased with a large effect size, and mean progress toward goals scores increased, also with a large effect size. Similarly, a smaller proportion of parents reported reliable improvement in psychosocial difficulties than impact on daily life or progress toward goals, whereas a larger proportion of parents reported no reliable change.

Change in psychosocial difficulties and impact on daily life showed fewer significant associations with change in clinician-reported functioning and parent-reported satisfaction with care at T2 than change in progress toward goals. Lower levels of psychosocial difficulties over treatment were associated with higher levels of satisfaction with care at T2. However, higher levels of progress toward goals over treatment were associated with higher levels of functioning over treatment and satisfaction with care at T2.

The relationships between change in progress toward goals with change in functioning and satisfaction with care at T2 were consistently stronger than the relationships between change in psychosocial difficulties or impact on daily life. Finally, change in progress toward goals was uniquely significantly associated with change in clinician-reported functioning, while change in psychosocial difficulties and impact on daily life were not, and clinicians reported higher levels of improvement in functioning for children and young people who made more progress toward achieving goals.

Findings of the present research may support findings from previous studies, where service users and clinicians have reported that idiographic measures are more capable than standardised measures of capturing relevant change for individuals (Bromley \& Westwood, 2013; Norman, et al., 2013; Pender, et al., 2013). Standardized measures may be more useful for comparing change in groups of patients and more amenable to aggregation; however, they may be perceived as lacking clinical utility as they may not reflect the specific difficulties of individual children (Bromley \& Westwood, 2013; Norman, et al., 2013; Pender, et al., 2013; Ruble, et al., 2012). 
In contrast it has been argued that the idiosyncratic nature of measures such as the GBO makes them less appropriate for comparison and more vulnerable to subjective interpretation, especially if performance targets are introduced (Bevan \& Hood, 2006; Law, 2011). Findings of the present research support the psychometric properties of the GBO, as with other goal-setting measures used in healthcare (Donnelly \& Carswell, 2002; Hurn, et al., 2006). In the present study, the internal consistency of the GBO was acceptable, suggesting that despite pertaining to different goals, ratings of progress toward goals may relate to the same underlying construct. Ratings of progress toward achieving goals set at the start of treatment may correspond to changes in patient activation: patients feel better able to selfmanage health conditions (Hibbard \& Gilburt, 2014) by working towards-and attainingpersonally meaningful goals. In addition, the reliable change index for the GBO in the present study was 2.45 points, suggesting that change greater than this is unlikely to be solely attributable to measurement error (Jacobson \& Traux, 1996). This may help clinicians interpret ratings of progress toward goals when monitoring the GBO of children, young people, and families.

Findings of the present research should be considered in the context of a number of limitations. First, data were not available on the content of goals, and unlike other measures (e.g. Goal Attainment Scaling; Kiresuk \& Sherman, 1968) other aspects of goals are not recorded in the GBO, such as relative importance and anticipated difficulty of goal attainment. Consequently, we cannot be certain that aggregating progress toward different goals represents the same latent factor, despite it fitting with our a priori theoretical explanations and the internal consistencies found (see Measures). Future research should continue to explore the psychometric properties of the GBO.

Second, goals can be set by the child or young person, parent, clinician, or ideally jointly agreed (Law, 2011). We were unable to explore this in the present study: future research should explore whether who sets goals influences change, interpretations, associations, and internal consistency. Similarly, child self-report data on psychosocial difficulties were not available for the present study. Children, parents, and clinicians may not necessarily agree on areas of difficulties and reasons for attending CAMHS (Yeh \& Weisz, 2001). Future research should explore whether progress toward goals shows the same pattern of associations with child-self reports as with parent- and clinician-reports.

Finally, the present research focussed on a sample of children and young people for whom relevant data were available from a wider dataset (see Participants and Procedure). This may limit the generalizability of findings to other children and young people seen by CAMHS, and future research should replicate the findings using larger, more representative samples. In line with expectations, psychosocial difficulties and impact on daily life showed less change than progress toward goals in the present study in terms of mean score change and reliable change. Change in psychosocial difficulties and impact on daily life was less strongly associated with change in clinician-reported functioning and satisfaction with care at T2 than change in progress toward goals, and only change in progress toward goals was uniquely associated with change in clinician-reported functioning over treatment.

Findings of the present research suggest that change in the GBO may be more relevant to change in functioning and satisfaction with care at T2 than in psychosocial difficulties and impact on daily life. Future research should continue to explore the GBO, what change means, and its psychometric properties. The present research suggests that idiographic measures may be more capable than standardised measures of capturing relevant change for individuals.

\section{References}

Attridge-Stirling, J. (2003). Development of methods to capture users' views of child and adolescent mental health services in clinical governance reviews London.

Austin, J. T., \& Vancouver, J. B. (1996). Goal constructs in psychology: Structure, process, and content. Psychological Bulletin, 120(3), 338-375. doi: 10.1037/00332909.120.3.338. 
Battle, C. C., Imber, S. D., Hoehn-Saric, R., Nash, E. R., \& Frank, J. D. (1966). Target complaints as criteria of improvement. American Journal of Psychotherapy, 20(1), 184-192.

Batty, M. J., Moldavsky, M., Foroushani, P. S., Pass, S., Marriott, M., Sayal, K., \& Hollis, C. (2013). Implementing routine outcome measures in child and adolescent mental health services: from present to future practice. Child and Adolescent Mental Health, 18(2), 82-87. doi: 10.1111/j.1475-3588.2012.00658.x

Bevan, \& Hood. (2006). What's measured is what matters: targets and gaming in the English public health care system. Public Administration, 84, 517-538. doi: 10.1111/j.14679299.2006.00600.x

Bickman, L., Kelley, S. D., Breda, C., de Andrade, A. R., \& Reimer, M. (2011). Effects of routine feedback to clinicians on mental health outcomes of youths: Results of a randomized trial. Psychiatric Services, 62(12), 1423-1429.

Bradley, J., Murphy, S., Fugard, A. J. B., Nolas, S. M., \& Law, D. (2013). What kind of goals do children and young people set for themselves in therapy? Developing a goals framework using CORC data. The Child and Family Clinical Psychology Review, 1, 8-18.

Bromley, C., \& Westwood, S. (2013). Young people's participation: views from young people on using goals. Clinical and Family Psychology Review, 1, 41-60.

Brown, A., Ford, T., Deighton, J., \& Wolpert, M. (2012). Satisfaction in Child and Adolescent Mental Health Services: Translating Users' Feedback into Measurement. Administration and Policy in Mental Health and Mental Health Services Research. doi: 10.1007/s10488-012-0433-9

Carlier, I. V. E., Meuldijk, D., Van Vliet, I. M., Van Fenema, E., Van der Wee, N. J. A., \& Zitman, F. G. (2012). Routine outcome monitoring and feedback on physical or mental health status: evidence and theory. Journal of Evaluation in Clinical Practice, 18(1), 104-110. doi: 10.1111/j.1365-2753.2010.01543.x

Chen, J., Ou, L., \& Hollis, S. (2013). A systematic review of the impact of routine collection of patient reported outcome measures on patients, providers and health organisations in an oncologic setting. BMC Health Services Research, 13(1), 211.

Cohen, J. (1988). Statistical Power Analysis for the Behavioral Sciences. New York: Erlbaum.

CORC. (2014a). Getting started - Snapshort approach, from http://www.corc.uk.net/joining/getting-started/getting-started-snapshot-approach/

CORC. (2014b). Homepage, from www.corc.uk.net

CORC. (2014c). Written evidence submitted by Child Outcomes Research Consortium (CORC)

(CMH0141),

from

http://data.parliament.uk/writtenevidence/committeeevidence.svc/evidencedocument/ health-committee/childrens-and-adolescent-mental-health-andcamhs/written/7632.html

Department of Health. (2010). Equity and excellence: Liberating the NHS. London.

Department of Health. (2012). IAPT three-year report: The first million patients. London.

Donnelly, C., \& Carswell, A. (2002). Individualized outcome measures: A review of the literature. . Canadian Journal of Occupational Therapy, 69(2), 84-94 doi: http://dx.doi.org/10.1177/000841740206900204

Goodman, R. (1997). The Strengths and Difficulties Questionnaire: a research note. J Child Psychol Psychiatry, 38(5), 581-586.

Goodman, R. (2001). Psychometric properties of the Strengths and Difficulties Questionnaire. Journal of the American Academy of Child \& Adolescent Psychiatry, 20, 1337-1345.

Goodman, R., Meltzer, H., \& Bailey, V. (1998). The strenghts and difficulties questionnaire: A pilot study on the validity of the self-report version. European Child \& Adolescent Psychiatry, 7, 125-130. doi: 10.1007/s007870050057

Green, H., McGinnity, A., Meltzer, H., Ford, T., \& Goodman, R. (2005). Mental health of children and young people in Great Britain, 2004. London: Palgrave. 
Hibbard, J., \& Gilburt, H. (2014). Supporting people to manage their health. An introduction to patient activation. London: The King's Fund.

Hurn, Kneebone, I., \& Cropley, M. (2006). Goal setting as an outcome measure: a systematic review. Clinical Rehabilitation, 20, 756-772.

Improvement, N. I. f. I. A. (2013). Standard NHS contract for acute services: schedule 18 part 2: Commissioning for Quality and Innovation (CQUIN). Retrieved from http://www.institute.nhs.uk/images/documents/wcc/PCT\%20portal/CQUIN\%20schem es/WM\%20-\%20Acute\%20BCH\%202010-11.pdf website:

Jacobson, N. S., \& Truax. (1991). Clincial signficance: a statistical approach to defining meaningful change in psychotherapy research. Journal of Consulting and Clinical Psychology, 59, 12-19.

Karoly, P. (1993). Goal systems: an organizing framework for clinical assessment and treatment planning. Psychological Assessment, 5(3), 273-280.

Kelley, S. D., \& Bickman, L. (2009). Beyond outcomes monitoring: measurement feedback systems in child and adolescent clinical practice. Current Opinion in Psychiatry, 22(4), 363-368. doi: 10.1097/YCO.0b013e32832c9162

Kiresuk, T. J., \& Sherman, R. E. (1968). Goal attainment scaling: A general method for evaluating comprehensive community mental health programs. Community Mental Health Journal, 4(6), 443-453. doi: 10.1007/BF01530764

Kluger, A. N., \& DeNisi, A. (1996). The effects of feedback interventions on performance: A historical review, a meta-analysis, and a preliminary feedback intervention theory. Psychological Bulletin, 119, 254-284.

Knaup, C., Koesters, M., Schoefer, D., Becker, T., \& Puschner, B. (2009). Effect of feedback of treatment outcome in specialist mental healthcare: Meta-analysis. The British Journal of Psychiatry, 195(1), 15-22.

Lambert, M. J., \& Shimokawa, K. (2011). Collecting client feedback. Psychotherapy, 48(1), 72-79. doi: $10.1037 / \mathrm{a} 0022238$

Lambert, M. J., Whipple, J. L., Hawkins, E. J., Vermeersch, D. A., Nielsen, S. L., \& Smart, D. W. (2006). Is It Time for Clinicians to Routinely Track Patient Outcome? A MetaAnalysis. Clinical Psychology: Science and Practice, 10(3), 288-301. doi: 10.1093/clipsy.bpg025

Law, D. (2011). Goals and Goal Based Outcomes (GBOs): some useful information. London: CAMHS Press.

Locke, E. A., \& Latham, G. P. (1990). A theory of goal setting and task performance. Englewood Cliff, $\mathrm{NH}$ : Prentice-Hall.

Maher, C. A., \& Barbrack, C. R. (1984). Evaluating the individual counseling of conduct problem adolescents: the goal attainment scaling method. Journal of School Psychology, 22, 285-297.

Malek, H., \& Joughlin, C. (2004). Mental health services for minority ethnic children and adolescents. London: Jessica Kingsley Publishers.

Moran, P., Kelesidi, K., Guglani, S., Davidson, S., \& Ford, T. (2011). What do parents and carers think about routine outcome measures and their use? A focus group study of CAMHS attenders. Clinical Child Psychology and Psychiatry, 17(1), 65-79. doi: $10.1177 / 1359104510391859$

National Quality Forum. (2013). Patient Reported Outcomes (PROs) in performance measurement. Washington, DC: National Quality Forum.

NHS. (2015). Governance Arrangements for Research Ethics Committees (GAfREC) Retrieved 13/03/15, 2015, from http://www.hra.nhs.uk/resources/research-legislationand-governance/governance-arrangements-for-research-ethics-committees/

Norman, S., Dean, S., Hansford, L., \& Ford, T. (2013). Clinical practitioner's attitudes towards the use of Routine Outcome Monitoring within Child and Adolescent Mental Health Services: A qualitative study of two Child and Adolescent Mental Health Services. Clinical Child Psychology and Psychiatry. doi: 10.1177/1359104513492348

Nunally, J. C. (1967). Psychometric theory. New York: McGraw-Hill. 
Pender, F., Tinwell, C., Marsh, E., \& Cowell, V. (2013). Evaluating the use of goal-based outcomes as a single patient rated outcome measure across CWP CAMHS: a pilot study. Child and Family Clinical Psychology Review, 1, 29-40.

Rey, J. M., Starling, J., Wever, C., Dossetor, D. R., \& Plapp, J. M. (1995). Inter-rater reliability of global assessment of functioning in a clinical setting. $J$ Child Psychol Psychiatry, 36, 787-792.

Ruble, L., McGrew, J. H., \& Toland, M. D. (2012). Goal attainment scaling as an outcome measure in randomized controlled trials of psychosocial interventions in autism. Journal of Autism and Developmental Disorders, 42(9), 1974-1983. doi: 10.1007/s10803-012-1446-7

Sapyta, J., Riemer, M., \& Bickman, L. (2005). Feedback to clinicians: Theory, research, and practice. Journal of Clinical Psychology, 61(2), 145-153. doi: 10.1002/jclp.20107

Shaffer, D., Gould, M. S., Brasic, J., Ambrosini, P., Fisher, P., Bird, H., \& Aluwahlia, S. (1983). A children's global assessment scale (CGAS). Archives of General Psychiatry, 40, 1228-1231.

Steiger, J. H. (1980). Tests for comparing elements of a correlation matrix. Psychological Bulletin, 87, 245-251. doi: http://dx.doi.org/10.1037/0033-2909.87.2.245

Whipple, J. L., \& Lambert, M. J. (2011). Outcome Measures for Practice. Annual Review of Clinical Psychology, 7(1), 87-111. doi: 10.1146/annurev-clinpsy-040510-143938

Wolpert, M., Deighton, J., De Francesco, D., Martin, P., Fonagy, P., \& Ford, T. (2014). From 'reckless' to 'mindful' in the use of outcome data to inform service-level performance management: perspectives from child mental health. BMJ Quality \& Safety. doi: 10.1136/bmjqs-2013-002557

Yeh, M., \& Weisz, J. R. (2001). Why Are We Here at the Clinic? Parent-Child (Dis) Agreement on Referral Problems at Out-Patient Treatment Entry. Journal of Consulting and Clinical Psychology, 69, 1018-1025. 